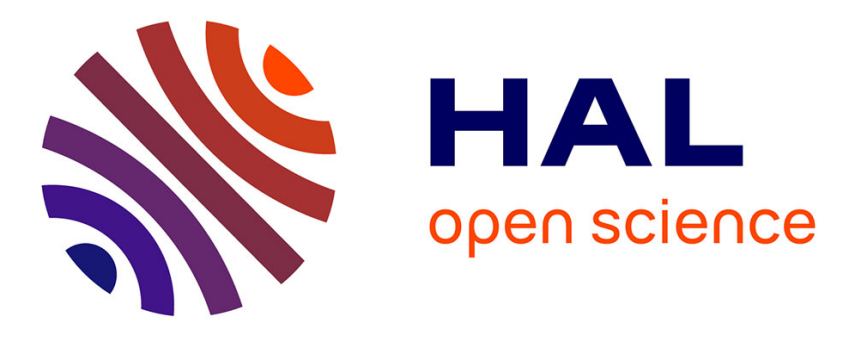

\title{
Multi-cell Coordination in Cloud RAN: Architecture and Optimization
}

Dora Boviz, Nivine Abbas, Gopalasingham Aravinthan, Chung Shue Chen, Mohamed Amine Dridi

\section{- To cite this version:}

Dora Boviz, Nivine Abbas, Gopalasingham Aravinthan, Chung Shue Chen, Mohamed Amine Dridi. Multi-cell Coordination in Cloud RAN: Architecture and Optimization. The international conference on wireless networks and mobile communications (WINCOM'16), Oct 2016, Fez, Morocco. hal01380756

\section{HAL Id: hal-01380756 https://hal.inria.fr/hal-01380756}

Submitted on 17 Oct 2016

HAL is a multi-disciplinary open access archive for the deposit and dissemination of scientific research documents, whether they are published or not. The documents may come from teaching and research institutions in France or abroad, or from public or private research centers.
L'archive ouverte pluridisciplinaire HAL, est destinée au dépôt et à la diffusion de documents scientifiques de niveau recherche, publiés ou non, émanant des établissements d'enseignement et de recherche français ou étrangers, des laboratoires publics ou privés. 


\title{
Multi-cell Coordination in Cloud RAN: Architecture and Optimization
}

\author{
Dora Boviz*, Nivine Abbas ${ }^{\dagger}$, Gopalasingham Aravinthan*, Chung Shue Chen*, Mohamed Amine Dridi* \\ *Nokia Bell Labs, Nozay 91620, France \\ E-mails: \{dora.boviz,gopalasingham.aravinthan, chung_shue.chen, mohamed_amine.dridi\}@ nokia-bell-labs.com \\ †Orange Labs and Télécom ParisTech, Université Paris-Saclay, France \\ E-mail: nivine.abbas@ telecom-paristech.fr
}

\begin{abstract}
Coordination between neighboring cells is intended to be implemented in future mobile networks, since it promises significant performance gains. Despite low-latency cooperation made possible by Cloud Radio Access Networks (C-RAN), practical feasibility and improvements brought to a real system were still to be evaluated. We define in this paper an architecture based on the abstraction and scalability provided by Software Defined Networking (SDN) enabling multi-cell coordination both on the uplink and downlink. We also evaluate gains offered by the proposed coordination algorithms under practical conditions. The described proof-of-concept platform shows not only why multi-cell cooperation is useful, but also how to make it happen.
\end{abstract}

Keywords-CoMP, Cloud-RAN, SDN, cooperation, interference management.

\section{INTRODUCTION}

As mobile network densification is a key solution enabling higher data rates in next generation $5 \mathrm{G}$ deployments, dealing with inter-cell interference (ICI) in cell-edge region becomes inevitable. Fortunately, the idea of centralizing at least a part of the baseband processing of several cells allows not only cost-saving but also multi-cell cooperation. Coordinated Multi-Point (CoMP) techniques [1], [2] already introduced in LTE require however centralized control over functions to realize cooperative processing in each cell. For this reason, we describe in the following a novel framework that associates centralized processing of Cloud Radio Access Network (C-RAN) to Software Defined Networking (SDN) providing an abstraction between baseband functions and coordination algorithms.

C-RAN enables low-latency cooperation between cells, since data between Baseband Units (BBUs) associated to different cells can be shared locally, without data transfer between distant cell sites. Combining multi-site reception and multi-user joint detection would enable significant spectral efficiency gains especially when dealing with high uplink (UL) traffic demand. However, to implement this feature we have to deal with several challenges such as synchronization, low RTT delay, fronthaul rate limitation and coordination among cells. A front-end architecture accommodating these constraints has been proposed in [3].

Despite being simpler to implement in C-RAN than its equivalent on the uplink, coherent Joint Transmission (JT) on the downlink (DL) [4] provides lower gains and high resource consumption in practical configuration [5]. In the literature, most of the studies are carried out around JT, which is considered as the most promising joint processing technique and thus little attention has been paid to Dynamic Point Blanking (DPB) [6]-[8], which seems to be a much simpler yet powerful technique compared to JT. Centralized coordination of multi-cell scheduling enables dynamic blanking in order to remove inter-cell interference received by user equipments (UEs) and thus improve overall throughput. We have selected DPB technique to be implemented in our C-RAN. Coordinated Multi-Point prototype platform offers better throughput to celledge users on the downlink.

Both coordination and joint reception/transmission require to exchange some data between the BBUs associated to different cells. Although they are placed in the same processing unit, they remain logically separated. In order to ensure scalability of our architecture, control plane is centralized and separated from data-plane traffic thanks to Software Defined Networking (SDN). It provides an interface between the BBUs to collect real-time data and possibly change their configuration, in order to apply joint optimization of network parameters for all of them while ensuring flexibility of the network size. We have also demonstrated that processing times offered by a SDN controller are compatible with RAN delay requirements in usual CoMP use cases.

$\mathrm{SDN}$ is identified, in addition to numerous other advantages, as an enabler of inter-cell interference coordination in $5 \mathrm{G}$ architecture described in [9]. In fact, controlling BBUs in a centralized way with low latency can also allow us to improve user association in heterogeneous access networks [10], realize multi-cell scheduling for CoMP [11], and improve dynamic function placement. The SDN controller keeps its initial function to act on fixed network routing that can make fronthaul and backhaul data transfer more reliable and efficient in terms of latency and bandwidth.

In the present work, we describe a flexible framework where the centralized BBU processing of C-RAN is linked to radio network intelligence applications through the abstraction level with SDN controller adapted to RAN. The main contribution of this work is to define an architecture where coordination algorithms for multi-cell joint processing can be executed given the practical constraints of a real-time RAN deployment. As specific use cases, we describe how coordination is performed, 
regarding both communication with the BBUs and its effect on the state of the RAN. Our prototype platform enables end-toend connectivity with enhanced Quality-of-Service (QoS) for cell edge users who would be affected by ICI without CoMP.

The remainder of the paper is organized as follows. In Section II, we present the architecture of the framework and its advantages for 5G RAN. Then, we present uplink and downlink coordination methods in Section III, followed by the verifications realized on the prototype platform that confirms the feasibility of the proposed solution in Section IV. We point out conclusions of this work and future research directions in Section V.

\section{Software Defined Cloud RAN Architecture}

\section{A. Dynamic PHY and MAC layers}

The Cloud-RAN architecture allows to split the base-band processing between distributed Remote Radio Heads (RRHs) located on the cell sites and the central processing unit (called BBU-pool) to which they are connected. Partial centralization of base-band processing of several cells to the BBU-pool has operational advantages, such as lower energy consumption, easier maintenance and robustness, but also numerous functional advantages e.g., low-latency data exchange between the BBUs. Moreover, base-band processing is independent from the computational hardware deployment, each function can be configured dynamically and instantiated in different places.

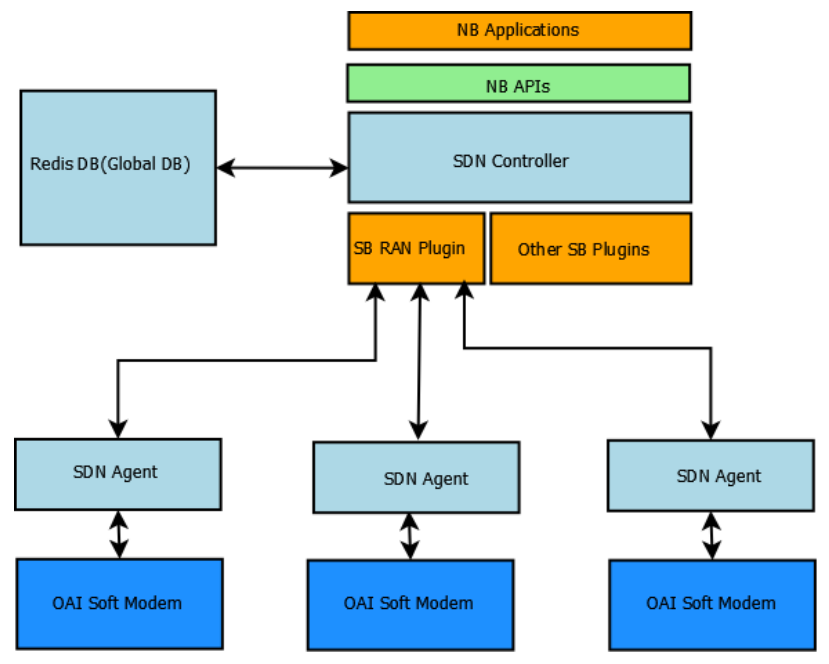

Fig. 1. Architecture proposed for scalable BBU control is C-RAN

To realize UL and DL CoMP processing aiming QoS improvement on the cell-edge, we propose an architecture where the functions required for joint processing are centralized (see [3]). Lower PHY functions can be placed in the RRH, but since our prototype platform does not aim to demonstrate function deployment and with a small-scale system performance is not affected by fronthaul limitation, we decided to execute all base-band processing in the BBU-pool to benefit fully from centralization advantages. In the following, BBU and eNodeB (eNB) are equivalent, since an eNB comprises all
BBU functions. The architecture depicted in Figure 1 makes the system scalable since the control of CoMP functions is centralized through the SDN controller and data exchange between the eNBs is necessarily controlled by it too. The controller can be placed in the BBU-pool server or in a remote location. Each eNB running in the same BBU-pool communicates individually with the SDN controller, sends and receives data related to its assigned cell. This data allows higher latency and generates less traffic than the one required for PHY multi-cell processing, which is shared inside the BBU-pool without coordination required from the controller. Selected RAN state measurements form PHY and MAC layer are extracted from a local database (DB) by a dedicated SDNagent and forwarded to the SouthBound (SB) interface of the controller. The role of the controller here is to provide abstraction between applications realizing CoMP coordination at the level of the scheduler and the eNBs.

\section{B. Interconnection between BBU-pool and SDN controller}

We have implemented the SouthBound (SB) protocol in the SDN controller for the abstraction of base stations using SCTP protocol due to its numerous advantages. For instance, message orientation in the protocol preserves the message boundaries where as in TCP the application has to define them. Furthermore, it ensures reliable message delivery without additional constraints and provides stronger (32 bits) end-toend checksum compared to TCP and UDP. Also, it is the legacy transport protocol used in message transfer between entities in mobile networks [12].

The SB protocol in the controller is a key element in bringing programmability to the RAN by

1) collecting necessary measurements, configuration, and neighbor information of each eNodeB, and

2) modifying/re-programming the functionality of each $\mathrm{eN}$ odeB by sending back new configuration parameters obtained from northbound (NB) applications.

In order to support the deployment of user coordination algorithm, the SDN controller has been extended both in Service Abstraction Layer and also in NB API modules since the controller supports only fixed network control by default. Since the YANG model based XML data storage [13] in the controller is not persistent to maintain long term network history, we introduced No-SQL database (Mongo DB, Redis, etc) connectivity to the SDN controller to bring persistency.

Each eNodeB is equipped with SDN-Agent to establish communication with the centralized controller. The SDNAgent module is built using the SCTP client libraries similar to the SB protocol that is built using SCTP server libraries. Since SCTP supports point to multi-point communication, any number of eNodeBs can be served by single SDN controller in parallel. Moreover, the SDN-Agent collects real-time measurements to be forwarded to the controller and also applies for example scheduling parameters received through the SB interface. 


\section{Northbound coordinator applications}

The main motivation behind the application of SDN approach to RAN is to abstract the entire multi-cell network intelligence to the BBUs that can use coordination algorithms as NB applications to benefit from joint processing possibilities offered by $\mathrm{C}-\mathrm{RAN}$ in order to enhance user experience. In our architecture, we have extended the NB APIs of the SDN controller to have necessary interfaces for NB applications (coordination algorithms) to retrieve required key performance indicators (KPIs) and configuration parameters as well as to re-configure the functioning of RAN using better parameters. In our architecture, NB applications interact with the external DB unit of the controller where set of tables have been created by the controller according to various level of RAN abstraction (neighbor information, configuration parameters, measurements per user, etc). During the operation, RAN service modules in the controller using the SB plug-in frequently updates the RAN inventory (RAN tables in the DB). NB APIs are built as HTTP REST (Representational State Transfer) full compliant APIs that facilitates the deployment of applications even in machines that are remote to the controller.

\section{User COORdinAtion Algorithms}

In this section, we describe the algorithms deployed on the NB interface of the controller, realizing the coordination of the users for UL and DL CoMP methods. The technical details are explained below.

\section{A. Uplink CoMP user selection}

1) Multi-cell multi-user detection: It is clear that installing RRHs more densely to improve throughput will also increase the regions where coverage overlaps among cells and intercell interference affects the received signals. Non-orthogonal multiple access (NOMA) scheme can be applied for the multicell uplink channel thanks to joint processing possible in the BBU-pool. Allocating all available orthogonal channel resources to the whole set of users allows, to achieve higher cell throughput, but in practice receiver complexity would be prohibitive. Scheduling a limited number of users on each subcarrier and applying multi-user detection techniques for them still improves spectral efficiency while detection complexity will be lower [14]. Due to full frequency reuse between cells, in a scenario with many UEs at the edge of two (or more) cells, the same subcarrier can be allocated to users in several cells who then mutually interfere. It is more efficient in this situation to use multi-user detection for them, thus CoMP coordination algorithm is especially relevant.

To illustrate the effect of ICI on a simple example, we define a system with two user equipments (UEs) transmitting on the same frequency resource and two receive RRHs each of them having 1 antenna (see Figure 2). The same observations stand for larger systems as far as the total number of receive antennas at all RRHs is greater or equal to the number of co-channel users. Uplink power allocation strategy of both users is assumed similar, since they are located almost at the same distance from the RRHs. Channel gains between the UEs and the RRHs are assumed independent and having the same statistics.

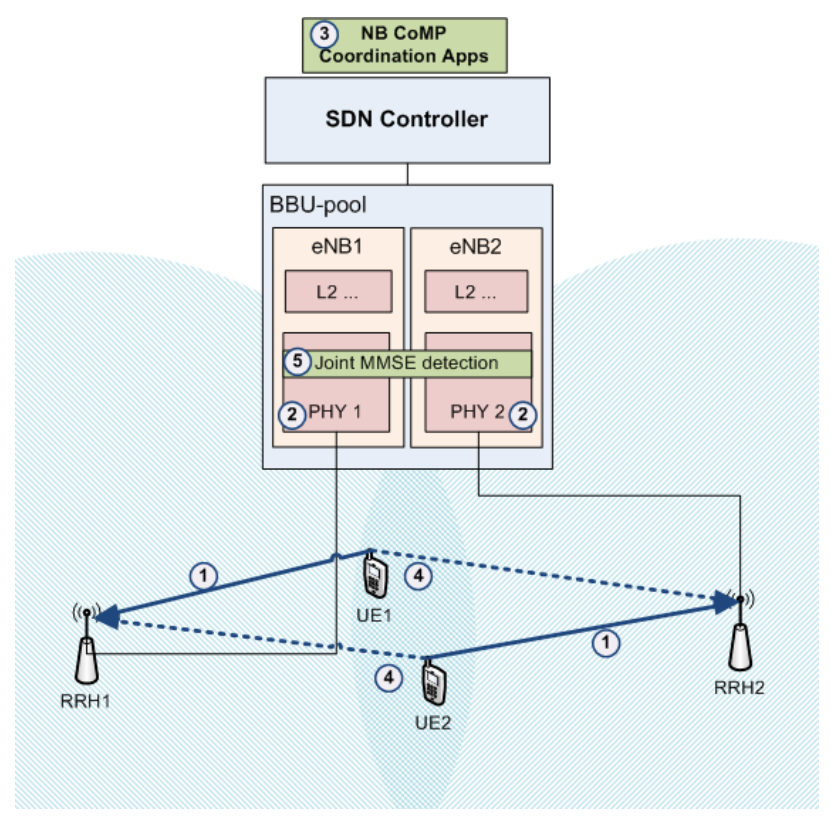

Fig. 2. Uplink joint detection architecture and coordination process

The received signal at RRH $j \in\{1,2\}$ can be written as follows:

$$
y_{j}=h_{1 j} x_{1}+h_{2 j} x_{2}+z_{j}
$$

where $h_{i j}, \forall i \in\{1,2\}$, is the channel gain between UE $i$ and RRH $j, x_{i}, \forall i \in\{1,2\}$, is the signal transmitted by UE $i$, and $z_{j}$ is the received AWGN having variance $\sigma_{z}^{2}$.

If single-user detection is performed independently for each uplink signal, the signal-to-interference-and-noise-ratio (SINR) of the received signal of both users would be very low because of the interfering signal having nearly the same power as the useful one. Consequently, accurate detection can be performed only by exploiting received signal from both RRHs. Maximum Ratio Combining (MRC) receiver is able to exploit antenna diversity but cannot overcome interference. Multi-user channel equalization and interference attenuation effect is offered by Minimum Mean Square Error (MMSE) detector exploiting both direct and interfering channel estimates contained in $\hat{\boldsymbol{H}}=\left(\begin{array}{ll}\hat{h}_{11} & \hat{h}_{21} \\ \hat{h}_{12} & \hat{h}_{22}\end{array}\right)$. In fact, MMSE detection isolates each user's signal from the overall received signal vector $\boldsymbol{y}=\left(y_{1}, y_{2}\right)^{T}$ (see equation (2) below) while also minimizing received interference power, thus the SINR is higher than that with SU detection.

$$
\hat{\boldsymbol{x}}=\left(\begin{array}{c}
\hat{x}_{1} \\
\hat{x}_{2}
\end{array}\right)=\boldsymbol{W}^{H} \boldsymbol{y} \text { with } \boldsymbol{W}=\left(\hat{\boldsymbol{H}} \hat{\boldsymbol{H}}^{H}+\sigma_{z}^{2} I_{2}\right)^{-1} \hat{\boldsymbol{H}}
$$

2) Selection of users requiring JD: Monitoring all scheduling decisions of eNBs or using a centralized scheduler to detect when users transmitting with similar power use the 


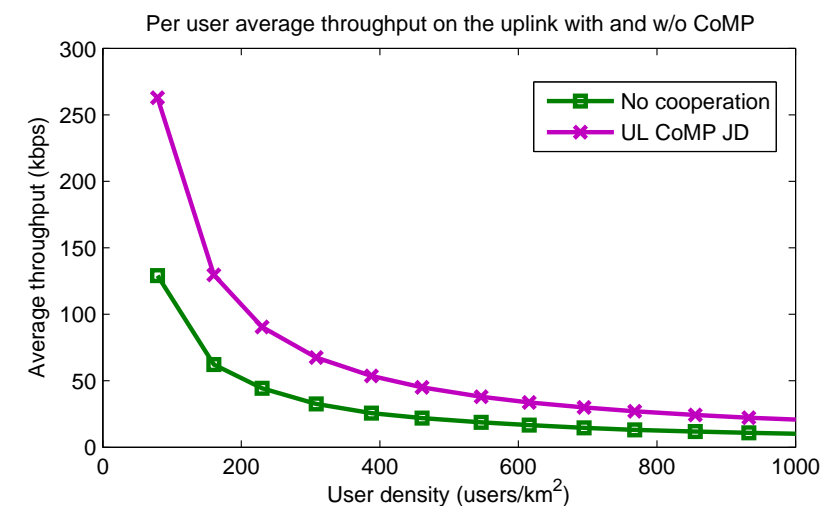

Fig. 3. Average throughput improvement with uplink JD

same wireless resource can be complex and it would introduce communication overhead between the eNBs and the controller. Furthermore, depending on the position of the users, the level of interference can happen to be very different. High ICI could be detected through SINR, but channel gains of interfering users are unknown, thus SINR cannot be computed. However, since low SINR results in higher error rates and lower effective throughput despite high received signal power, monitoring these elements can indicate the presence of high ICI. We chose to send regularly the above cited information to the UL CoMP coordinator which can then take the decision to apply multicell detection for users suffering from ICI. The controller sends then instructions to their respective eNBs to schedule them on the same physical resource and enable functions realizing joint detection in their physical layer receive processing.

We have evaluated the performance improvement enabled by UL JD for cell-edge users affected by ICI. Figure 3 depicts the variation of average user throughput with different cell loads. We simulated uplink transmission for a cell with omnidirectional antenna surrounded by 8 other cells. 16-QAM modulation scheme and $1 / 3$ coding rate are used and power allocation strategy is assumed to be similar in all cells. We observe that average throughput is around 2 times higher when JD is used, since significant degradation of cell-edge qualityof-service is resolved thanks to multi-cell cooperation.

3) Data sharing between BBUs: To enable multi-cell MMSE detection for every user transmitting on the same frequency resource, on one hand channel estimates between every UE and every RRH need to be computed and shared, on the other hand received signals also have to be made available to every eNB processing a cell involved in the joint detection scheme.

In LTE-Advanced, cyclic shifting of Demodulation Reference Signals (DMRS) is defined to be able to estimate the channel of up to 12 co-channel users. Cyclic shift values have to be controlled by the coordination algorithm, which then activates multi-user channel estimation in every eNB for cyclic shifts that are used with the given number of UEs. Once channel estimates are computed by each eNB for every user, they are forwarded to a common function computing the whole
MMSE matrix. To isolate the signal of each user, one line of the Hermitian of the MMSE matrix is multiplied by the vector of signals received by the whole set of RRHs that are shared between the eNB processes instantaneously, since they are running in the same server.

4) UL joint detection coordination algorithm: To summarize the algorithm realizing the coordination between different cells processed in the same BBU-pool required by multi-cell multi-user detection, we describe each step of it. The number of each step on Figure 2 represents the main functional block related to it.

(1) Each UE transmits towards the RRH of the cell to which it is associated and single-user detection is performed in the BBU (eNB).

(2) After PHY processing, error rates and received signal characteristics (modulation order, received power) are sent to the coordinator.

(3) Coordination algorithm detects high ICI and enables multi-user detection. It sends to the eNBs scheduling constraints for users involved in JD and instructions to activate joint detection functions (i.e., multi-user channel estimation, MMSE matrix computation and MMSE equalization).

(4) UEs transmit according to new parameters. (They are intentionally scheduled on the same wireless resource, thus create inter-cell interference.)

(5) Multi-cell joint MMSE detection is realized. Error rate is decreased w.r.t. previous transmission with SU detection and effective throughput is improved.

\section{B. Downlink multi-cell dynamic point blanking}

1) DPB concept: As interference is still the main limiting factor in cellular networks, several new approaches and features are being considered as key elements to cope with interference in future mobile networks. The main motivation for such new features is to increase the cell-edge user throughput and data rate coverage. In this context, DPB is considered as a promising technique to satisfy higher requirements in terms of capacity and cell edge user throughput. The main purpose of

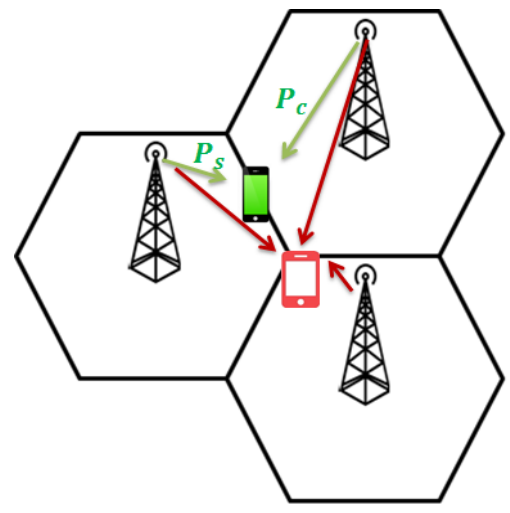

Fig. 4. Cell edge users strongly interfered by neighboring cells.

this concept is to help users at the cell edge (see Figure 4) who 
are suffering from a lot of interference from the neighboring cells through multi-cell coordination, mainly the serving cell and the interfering neighboring cells. These cells coordinate between each other in order to reduce interference to the socalled CoMP user, that is the user at the cell-edge affected by the interference.

DPB consists in identifying and dynamically muting the principal interferer(s) to the UEs. By muting the dominant interferer, the SINR of the UE could be significantly improved as dominant interferers may represent the majority of the whole interference.

Each cooperating cell $c \in \mathbb{C}_{u}$, where $\mathbb{C}_{u}$ is the set of cooperating cells of the considered CoMP user $u$, is muted. The instantaneous perceived SINR of user $u$ is then given by:

$$
S I N R_{u}(t)=\frac{P_{u, s}(t)}{\sum_{i \neq c \in \mathbb{C}_{u}} P_{u, i}(t)+N}
$$

where $P_{u, x}(t)$ is the power received by user $u$ from cell $x$ at time $t$. The instantaneous data rate $r_{u}(t)$ depends on $S I N R_{u}(t)$, and can be computed using LTE CQI tables.

2) Cooperating cell definition: As only some neighboring cells should cooperate in order to serve a given cell-edge user $u$, a definition of a cooperating cell is needed. Usually, this is done based on the averaged RSRP (reference signal received power), see [4], [15], [16]. When the difference between the average power received from the neighboring cell, denoted by $\bar{P}_{u, c}$, and that received from the serving cell, denoted by $\bar{P}_{u, s}$, is less than a given predefined threshold $\delta P$ (see Figure 4), the neighboring cell is defined as a cooperating cell for the considered user:

$$
\left|\bar{P}_{u, s}(t)-\bar{P}_{u, c}(t)\right|<\delta P .
$$

A neighboring cell fulfilling this condition can cooperate. Thus, the set of cooperating cells $\mathbb{C}_{u}$ of user $u$ is updated over time based on long-term UE power measurement, it is not expected to change over time as long as the location of the UE does not change.

3) Scheduling: Consider in each cluster, that is the set of cells that are allowed to cooperate between each other, a central scheduler that has knowledge of the CSI/CQI information of all users being served within the cluster. In this case, the simplest scheduling strategy, treating CoMP users and nonCoMP users equally, is achieved through the PF (proportional fair) iterative scheduler, described as follows:

The PF strategy selects the user with the highest instantaneous data rate relative to its mean data rate, measured in previous TTI (transmit time interval): $r_{u}(t) / \bar{R}_{u}(t)$.

This scheduler requires at most $|A|$ iterations in order to make scheduling decisions for all cells taking part in the cluster, as at most $|A|$ users can be scheduled in a given cluster of size $|A|$. This may happen when all scheduled users are non-CoMP users.

Note that this is a sub-optimal scheduling strategy. An optimal scheduling strategy, that consists in formulating an optimization problem, would provide higher performance than the above iterative scheduler but it will be at the cost of

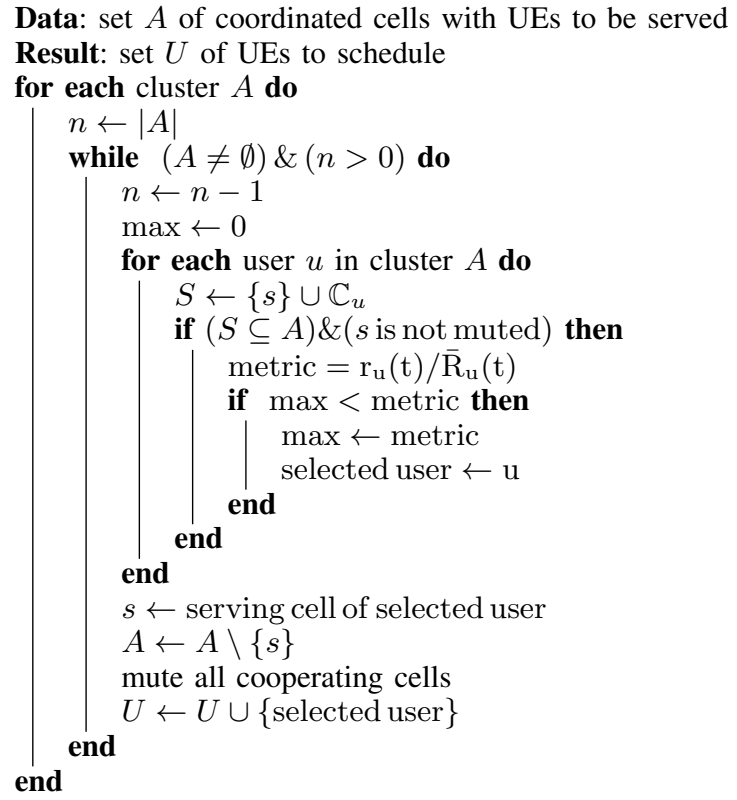

Algorithm 1: Centralized PF iterative scheduling for DPB.

higher complexity. Besides, this would require to exchange an enormous amount of signaling information

4) System level simulations: We have evaluated the performance of DPB under the described PF iterative scheduling strategy, by system-level simulations based on the LTE technology. We consider 21 hexagonal cells formed by 7 tri-sector sites (a reference site surrounded by 6 interfering sites). Both eNodeB and mobile devices are equipped with 2 antennas. Spatially correlated shadowing maps for different sites are generated each second, with time correlation. Each TTI (of 1 ms), 4 spatially correlated fading complex values are generated for each eNodeB to mobile device pair. The main parameters are summarized in Table I.

\begin{tabular}{|c|c|}
\hline Network topology & Macro cells only \\
\hline Environment & Urban \\
\hline Context & outdoor \\
\hline Inter-site distance & $500 \mathrm{~m}$ \\
\hline PathLoss & ITU Model \\
\hline Shadowing std & $4 \mathrm{~dB}$ \\
\hline Shadowing correlation distance & $30 \mathrm{~m}$ \\
\hline Radio access technology & LTE \\
\hline Number of tx/rx antennas & MIMO $(2 \times 2)$ \\
\hline Number of streams & MRC \\
\hline Receiver & $3 \mathrm{GPP}$ \\
\hline Codebook & $2 \mathrm{GHz}$ \\
\hline Carrier frequency & $10 \mathrm{MHz}$ \\
\hline Bandwidth & $\sigma=1.25 \mathrm{MBytes}$ \\
\hline Mean file size & 100 minutes $=6 \times 10^{6}$ TTI \\
\hline Simulation time & \\
\hline &
\end{tabular}

TABLE I

SiMULATION SETTING.

Traffic consists of file transfers only. Flows arrive according to a Poisson process with uniform spatial distribution. File sizes are generated from an exponential distribution with mean $\sigma$ (MBytes). Scheduling decisions are taken according 
to Algorithm 1. We simulate $6 \times 10^{6}$ TTI and we estimate the mean user throughput as the ratio of the mean flow size to mean flow duration, as given by Figure 5(a) . We study the case without coordination under classical PF scheduler as well as the case of DPB for different power threshold values $\delta P: 3 \mathrm{~dB}, 6 \mathrm{~dB}, 9 \mathrm{~dB}, 12 \mathrm{~dB}, 15 \mathrm{~dB}$ and $18 \mathrm{~dB}$ under $\mathrm{PF}$ iterative scheduler explained in Algorithm 1. We evaluate also in each case the mean number of users per eNodeB (see Figure 5(c)) and the relative throughput gain when applying DPB compared to the case without coordination, with respect to the offered traffic per eNodeB (see Figure 5(b)). All cells are allowed to cooperate between each other. We limit the number of cooperating cells for one user to 3 cells, including the serving cell.

We can observe that the relative gain increases with load. At low load, the gain brought by the elimination of the interference due to the muting of interfering cells is relatively small. This is due to the fact that when neighboring cells are slightly loaded, they are naturally muted most of the time and thus cooperation is likely to be less triggered. When the network becomes more loaded, interference between different cells becomes more important. In this case, the blanking of interfering cells at the right moment, when the scheduled user is strongly affected by the generated interference, seems to bring interesting gains. We observe that gains go up to $150 \%$. Furthermore, gains increase with $\delta P$ up to $\delta P=12 \mathrm{~dB}$ and then start to decrease.

The mean number of users in each cell increases much more slowly compared to the case where DPB is not applied. This technique seems to be promising as it is simple and it brings significant gains even under a sub-optimal simple scheduling strategy. However, in order to achieve the maximum gains an optimal scheduler must be used.

\section{TESTbED PERFORMANCE EVALUATION}

In order to confirm the possibility of realizing RAN configuration through the architecture that we are proposing, we have evaluated communication latency between the global DB connected to the controller and the eNB agent. It gives the delay under which new measurements are made available to the NB applications which then execute their respective algorithms and update their output parameters. Since algorithms are designed to perform efficiently a specific task, their execution time is very low. In the downstream direction, data transit delay is similar since the same SouthBound protocol is used.

Typical transit durations are observed to range from 0.4 ms to $0.7 \mathrm{~ms}$, meaning that one can expect to update eNB state $2 \mathrm{~ms}$ after having captured new measurements. If we assume that the cooperation scheme is efficient if up-to-date parameters are applied at least $50 \%$ of a channel coherence period, when we provide new values after every subframe of $1 \mathrm{~ms}$, the minimal channel coherence time when coordination is useful is around $6 \mathrm{~ms}$. In terms of mobility, it confirms that multi-cell coordination can be efficiently applied up to

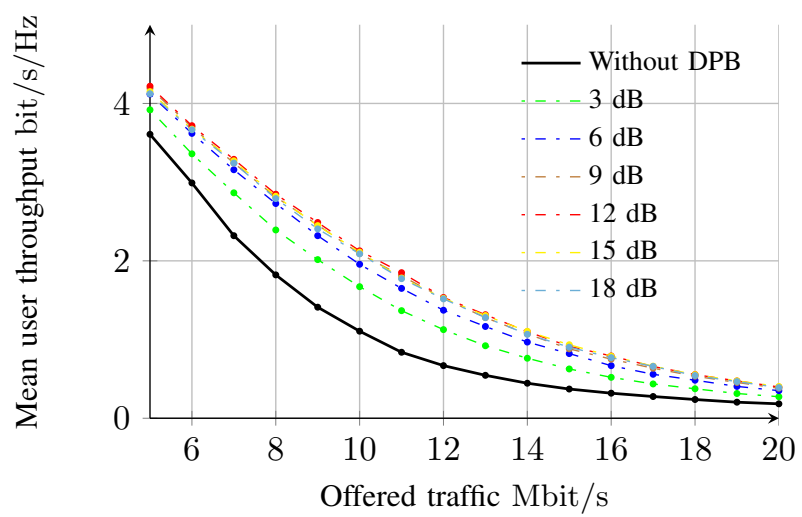

(a) Mean user throughput.

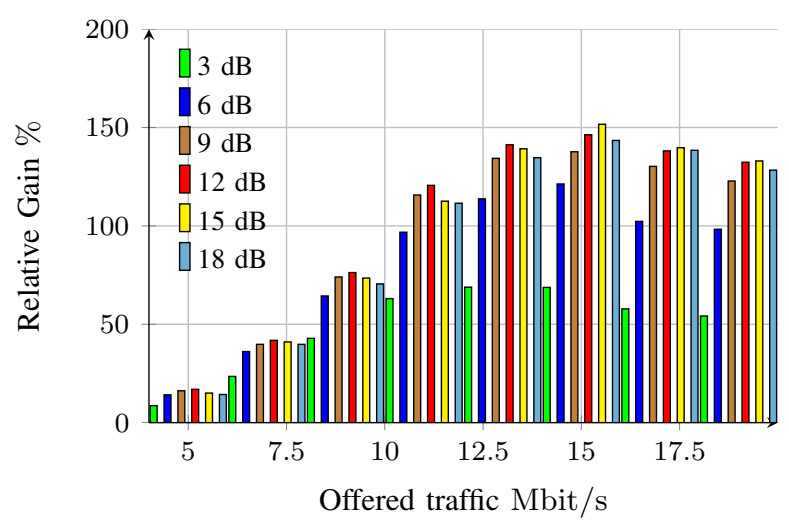

(b) Relative gains.

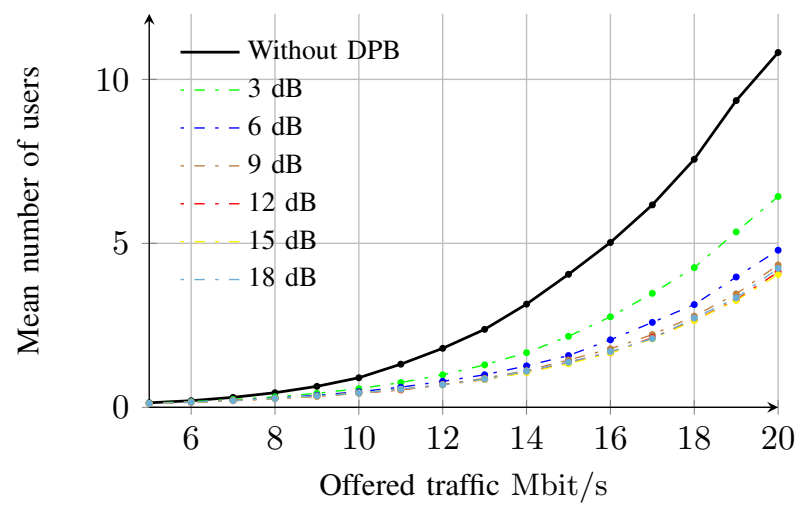

(c) Mean number of users per cell.

Fig. 5. Throughput performance obtained by system-level simulation for dynamic clustering DPB.

$300 \mathrm{~km} / \mathrm{h}$ of user velocity. This could support users with high mobility.

\section{Conclusion And Perspectives}

Significant gains by CoMP techniques under ideal conditions are observable. However, defining an explicit analytical model for practical systems and evaluating their performance with respect to realistic implementation constraints is still an open problem. As a conclusion of the present work, we have confirmed that UL JD and DL DPB can be deployed in future mobile networks using the scalable architecture that we have 
presented above. Our proof-of-concept prototype demonstrates not only the feasibility of the proposed architecture but also gains provided by multi-cell cooperation in C-RAN. CoMP coordination use cases are integrated in a scalable C-RAN framework that can accommodate various RAN enhancement applications thanks to the abstraction provided by the SDN controller. Results of extensive simulations are confirmed, though in a small size system, but in an environment ready to be deployed. In a future extension, adding dynamic function placement and fronthaul latency control NB applications could provide a more complete RAN control realization through SDN and enhanced user experience.

\section{ACKNOWLEDGMENT}

This work was supported in part by the ANR project IDEFIX under the grant number ANR-13-INFR-0006. A part of the work was carried out at LINCS (www.lincs.fr).

\section{REFERENCES}

[1] 3GPP TR 36.913, "Requirements for Further Advancements for EUTRA (LTE-Advanced)."

[2] R. Irmer, H. Droste, P. Marsch, M. Grieger, G. Fettweis, S. Brueck, H. Mayer, L. Thiele, and V. Jungnickel, "Coordinated multipoint: Concepts, performance, and field trial results," IEEE Communications Magazine, vol. 49, no. 2, pp. 102-111, 2011.

[3] D. Boviz and Y. El Mghazli, "Fronthaul for 5G: low bit-rate design enabling joint transmission and reception," in IEEE Globecom, December 2016.

[4] S. Brueck, L. Zhao, J. Giese, and M. A. Amin, "Centralized Scheduling for Joint Transmission Coordinated Multi-Point in LTE-Advanced," in Proc. ITG/IEEE Workshop on Smart Antennas, , Bremen, Germany, 2010.

[5] A. Khlass, T. Bonald, and S. Elayoubi, "Flow-level performance of intrasite coordination in cellular networks," in IEEE WiOpt, Tsukuba Japan, 2013, pp. 216-223.
[6] G. Morozov, A. Davydov, and I. Bolotin, "Performance evaluation of dynamic point selection CoMP scheme in heterogeneous networks with FTP traffic model," in 4th International Congress on Ultra Modern Telecommunications and Control Systems, St. Petersburg, Russia, 2012, pp. 922-926.

[7] H. Wang, X. Tao, and P. Zhang, "Adaptive modulation for dynamic point selection/dynamic point blanking," IEEE Communications Letters, vol. 19, no. 3, pp. 343-346, 2015.

[8] M. Li, X. Yun, S. Nagata, and L. Chen, "Power allocation of dynamic point blanking for downlink CoMP in LTE-Advanced," in International Conference on Wireless Communications and Signal Processing, Hangzhou, China, 2013, pp. 1-5.

[9] J. F. Monserrat, H. Droste, . Bulakci, J. Eichinger, O. Queseth, M. Stamatelatos, H. Tullberg, V. Venkatkumar, G. Zimmermann, U. Dötsch, and A. Osseiran, "Rethinking the mobile and wireless network architecture: The METIS research into 5G," in European Conference on Networks and Communications (EuCNC), June 2014, pp. 1-5.

[10] A. Gopalasingham, L. Roullet, N. Trabelsi, C. S. Chen, A. Hebbar, and E. Bizouarn, "Generalized software defined network platform for radio access networks," in IEEE Consumer Communications and Networking Conference, 2016.

[11] D. Boviz, A. Gopalasingham, C. S. Chen, and L. Roullet, "Physical layer split for user selective uplink joint reception in SDN enabled CloudRAN," in Australian Communication Theory Workshop, January 2016.

[12] L. Ong and J. Yoakum, "RFC 3286, An introduction to the stream control transmission protocol (SCTP)," http://www.faqs.org/rfcs/rfc3286.html, 2002.

[13] A. Lindem, L. Berger, D. Bogdanovic, and C. Hopps, "Network Device YANG Organizational Models," Aug. 2016, work in Progress. [Online]. Available: https://tools.ietf.org/html/ draft-rtgyangdt-rtgwg-device-model-05

[14] M. Al-Imari, P. Xiao, M. A. Imran, and R. Tafazolli, "Uplink nonorthogonal multiple access for $5 \mathrm{G}$ wireless networks," in 11th International Symposium on Wireless Communications Systems (ISWCS), Aug 2014, pp. 781-785.

[15] M. Sawahashi, Y. Kishiyama, A. Morimoto, D. Nishikawa, and M. Tanno, "Coordinated multipoint transmission/reception techniques for LTE-advanced," IEEE Wireless Commun., vol. 17, no. 3, pp. 26-34, 2010.

[16] B. Mondal, E. Visotsky, T. A. Thomas, X. Wang, and A. Ghosh, "Performance of downlink comp in LTE under practical constraints," in IEEE International Symposium on Personal, Indoor and Mobile Radio Communications, Sydney, Australia, 2012, pp. 2049-2054. 